\title{
Introduction: The Otto Scherzer Special Issue on Aberration-Corrected Electron Microscopy
}

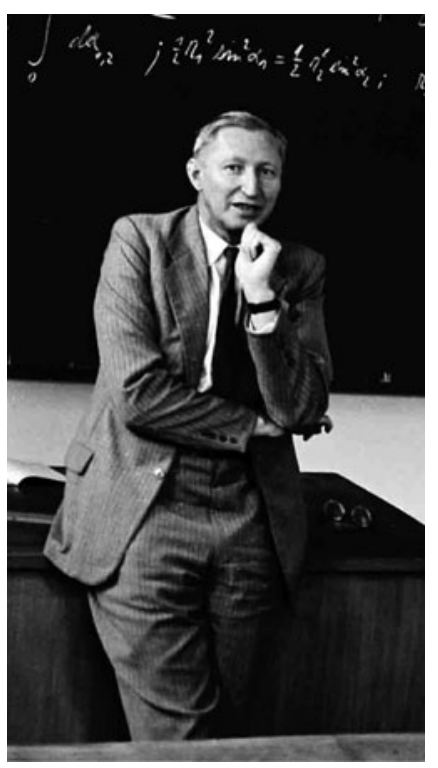

Image of Otto Scherzer (courtesy of Dieter Typke)
The year 2009 marked the centenary of the birth of Otto Scherzer, one of the early pioneers of electron microscopy. Scherzer was the originator of the famous microscopy theorem that the spherical and chromatic aberrations of rotationally symmetric electron lenses were unavoidable. In honor of this centennial occasion, we organized a special memorial symposium during Microscopy \& Microanalysis 2009, which was held in Richmond, Virginia, in late July. The introductory talks of the symposium presented a fascinating mix of firsthand accounts about working with Scherzer in Darmstadt and descriptions of the correction concepts and the early corrector prototypes that emerged from his group. Placed in this historical context, the latest advances in aberration correction for scanning and fixedbeam instruments that were presented in this symposium were all the more impressive and conveyed a vivid sense of history in the making. Applications of aberration correction to a broad range of materials were also highlighted in platform and poster presentations. This special issue of Microscopy and Microanalysis contains refereed contributions from the work presented at the symposium and thus provides a representative overview of the recent emergence of aberration-corrected electron microscopy (ACEM) and some of the prospects and challenges for this burgeoning field.

Spherical aberration is well known as a focusing defect that is inherent to circularly symmetric electron lenses, and it prevents off-axis electrons from all being focused to the same point. Aberration correction makes it possible to "tune" spherical aberration by making its value vanishingly small, or even negative. The latest aberration correctors take care of aberrations up to the fifth order, and these have succeeded in improving the resolution limits of the transmission electron microscope (TEM) to about $50 \mathrm{pm}$ for both fixed-beam and scanning-beam TEMs. Aberration correction can be achieved in several different ways. Online approaches in either fixed-beam TEM or scanning-beam STEM follow the principles outlined by Scherzer and use hardware corrector systems installed on the instrument, whereas off-line correction techniques use special software programs to reconstruct off-axis electron holograms or exit-surface wave functions. The online correctors involve various combinations of multipole elements that overcome the symmetry constraints recognized in Scherzer's original 1936 theorem, and the success of these correctors is an exciting development that has attracted much attention. Many striking examples of aberration-corrected TEM and aberration-corrected STEM images can be found elsewhere in this special issue. The prospects seem excellent for exciting times ahead for both the microscopy and materials communities using the latest generation of aberration-corrected instruments. Otto Scherzer would surely have been delighted to see the fruits of aberration correction finally being realized!

Guest Editors

David J. Smith and Uli Dahmen 